\title{
La dengue, maladie complexe
}

\author{
Éric Daudé ${ }^{1}$, Alain Vaguet ${ }^{2}$, Richard Paul ${ }^{3}$ \\ 1 Géographe, CNRS, Centre de Sciences humaines, UMIFRE 20 (CNRS/MAE), 110011 Delhi, Inde \\ 2 Géographe, CNRS, UMR 6266 IDEES (CNRS/Université de Rouen), 76821 Rouen, France \\ 3 Épidémiologue, Institut Pasteur, URA 3012 (CNRS/Institut Pasteur), 75724 Paris, France
}

La diffusion et l'émergence des risques sanitaires constituent une préoccupation très présente dans les discours actuels sur la mondialisation et les changements climatiques. La dengue y occupe une place de choix depuis qu'elle a été diagnostiquée sporadiquement aux portes de l'Europe et que son vecteur fréquente une part croissante de nos territoires nationaux. Cette question souvent traitée en termes de causalité trouve dans cet article une remise en perspective extrêmement utile, montrant que le risque ne naît pas d'une succession d'événements mais bien d'une articulation complexe de facteurs. En cela, les auteurs nous proposent d'interpréter la variabilité de l'expression de la maladie comme le produit aussi bien d'interactions à l'interface natures/sociétés que d'un dialogue entre disciplines, remettant en cause tout déterminisme dans la dynamique de ce risque sanitaire.

La Rédaction

Mots-clés :

dengue ;

système complexe ;

modélisation ;

simulation

\begin{abstract}
Résumé - La dengue est une infection virale à transmission vectorielle qui touche, pour l'instant, essentiellement les pays de la zone tropicale et subtropicale. Nous développons un cadre systémique et complexe pour l'étude de cette maladie, qui permet à la fois de décomposer ses différentes entités - hôte, vecteur, virus, environnement - tout en affirmant le poids de leurs interactions dans ses dynamiques potentielles. L'étude de cette complexité systémique s'enrichit d'un cadre méthodologique qui permet d'intégrer dans un même modèle des dynamiques biologiques et sociales, dans des environnements changeants. Ce cadre de recherche implique de nouvelles pratiques, il rend indispensable une collaboration étroite des sciences sociales, environnementales, biologiques et informatiques, sans oublier l'implication des populations et des acteurs locaux de santé publique.
\end{abstract}

Keywords:

Dengue;

complex systems; multiagent simulation

\begin{abstract}
Dengue, a complex disease. Dengue is a widespread mosquito-borne viral infection in tropical and subtropical countries causing an estimated 500 million infections per year of which more than 20,000 result in severe disease. Since the 1970s dengue has spread and become endemic in more than 100 countries and there is concern that dengue will spread into temperate regions. The peridomestic niche of the primary mosquito vector, Aedes aegypti, results in dengue being a largely urban and periurban disease where the high population densities cause major epidemics. Although a number of risk factors have been identified at different spatial scales of analysis, their general relevance is debatable. Very local scale environmental factors can determine both the abundance of mosquitoes and their ability to transmit the virus. Thus the environmental complexity of urban settings coupled with heterogeneity in human population density yield potentially complex interactions in highly differentiated local conditions that are difficult to assess. We are developing a complex framework to enable a more tractable understanding of dengue epidemiology. This framework enables the disentanglement of actors, host, vector, virus and environment, all the while asserting the dynamic contribution of their interactions. The study of this complexity is enriched by a methodological framework that integrates the biological and social dynamics in changing environments in a single model. The first section of the article describes the complexity of dengue epidemiology. The second section proposes multiagent spatial modelling as a methodological framework with which to integrate this complexity.
\end{abstract}

Auteur correspondant : E. Daudé, eric.daude@univ-rouen.fr 


\section{Explosions épidémiques d'une maladie " réémergente"}

La dengue, maladie à transmission vectorielle, est devenue une préoccupation majeure de santé publique internationale (Guzman et al., 2010). Près de $40 \%$ de la population mondiale, principalement situés dans les zones tropicales, seraient à risque d'infection selon l'OMS (2012a). Les estimations font état de 50 à 400 millions d'infections chaque année dans les pays endémiques, générant plus de 10000 décès (Bhatt et al., 2013). Maladie réémergente à partir des années 1970, son évolution à l'échelle mondiale est liée à d'importants changements sociétaux tels que l'urbanisation croissante et pas toujours maîtrisée (Gubler, 2011), l'absence ou le relâchement des politiques de santé publique (PAHO, 1997) et l'augmentation des flux de personnes et de biens (Gubler, 1998). Le changement climatique global conduit également à poser la question de l'extension des zones géographiques des moustiques vecteurs (Hales et al., 2002) et l'augmentation de la saison de transmission dans les zones où circule actuellement le virus de la dengue (Deubel et Rodhain, 1999).

Le moustique Aedes aegypti est le vecteur le plus compétent pour la transmission du virus à l'homme (Rodhain et Rosen, 1997). Une fois contaminé, l'homme devient temporairement porteur du virus et peut contaminer de nouveaux moustiques. La grande majorité des infections dues au virus de la dengue se caractérisent cependant par l'absence de symptôme. Ceci limite potentiellement les effets des mesures de contrôle de l'épidémie, notamment lorsque celles-ci sont déclenchées à la seule déclaration des cas, donc symptomatiques (Ooi et al., 2006). Si le contrôle de la maladie passe par la mise au point d'un vaccin, solution envisagée à long terme (Sabchareon et al., 2012), sa gestion à court et moyen terme passe par une surveillance de l'environnement, par le contrôle du moustique vecteur et par l'implication dans cette lutte des populations exposées au risque épidémique (Gubler et Kuno, 1997).

Le cycle de transmission de la maladie et sa diffusion géographique impliquent la coexistence du virus, d'une population d'hôtes et de vecteurs dans des profils environnementaux spécifiques. C'est donc les composants hôte/vecteur/virus/environnement et leurs interactions qu'il faut étudier pour comprendre le système de la dengue. Les dynamiques de ces composants sont influencées par différents facteurs dont l'importance dans l'évaluation du risque est relative aux échelles de temps et d'espace d'estimation de ce risque. S'agissant de la progression du risque vectoriel par exemple, le changement climatique et la hausse des températures sont des facteurs qui sont mis en avant à l'échelle mondiale (Descloux et al., 2012). Mais en l'état des connaissances, la variabilité des précipitations et des tempéra- tures ne semblent pas discriminantes à l'échelle intraurbaine d'un épisode épidémique (Rogers et Randolph, 2006), plutôt déterminé par le poids de contributions sociales et environnementales hétérogènes.

L'objectif de cet article est de montrer la complexité de la dengue. La première partie commence par la description de ses composants et de leurs interactions. L'absence de consensus sur les facteurs de risques épidémiques nous conduit ensuite à discuter les moyens de son observation, à différentes échelles, et à aborder la question de sa modélisation informatique. Il s'agit de modèles comportementaux, où l'on mobilise les connaissances sur les comportements des vecteurs et des hôtes pour produire, via la simulation, des données statistiques soumises aux regards critiques des experts du domaine. Cette modélisation permet de tester de nombreuses hypothèses et d'explorer des scénarios de propagation. Il ne s'agit pas ici de présenter un modèle en particulier, mais bien une méthode et une série de questions à laquelle celle-ci est susceptible d'apporter de nouvelles réponses.

\section{Complexité des maladies à transmission vectorielle : la dengue}

Les géographes ont largement contribué aux études des effets des contextes de vie sur les risques sanitaires, avec la définition des aires pathogènes et des paysages épidémiologiques (Picheral, 1983 ; Kitron, 1998). Une contribution importante pour la compréhension des mécanismes épidémiques est de montrer par exemple qu'une faible exposition au risque vectoriel peut malgré tout s'accompagner de bouffées épidémiques, du fait des mobilités des populations et des niveaux hiérarchisés de centralité des lieux (Handschumacher et Hervouet, 2004). Les modalités d'usages du sol, les fluctuations climatiques, les migrations humaines, les modes de vie des habitants ou encore l'urbanisation sont ainsi des facteurs reconnus de situations épidémiques. L'enjeu est alors de montrer qu'une approche systémique et complexe de la dengue permet de penser la question du risque à différentes échelles biologiques, d'espace et de temps.

\section{Les entités du système de la dengue}

Dans les pages qui suivent, nous proposons une synthèse partielle des connaissances sur les vecteurs, les hôtes, le virus et l'environnement. Les liens qui en font un système et les dynamiques qui en résultent forment la dimension complexe.

\section{Le vecteur : Aedes aegypti}

Le principal vecteur de la dengue est un moustique synanthropique, Ae. aegypti et secondairement Ae. albopictus, qui vit pour l'essentiel en milieu urbain et 
périurbain (Vezzani et al., 2005). Chez Aedes seule la femelle pique, de préférence en début et fin de journée, les repas sanguins lui procurant les nutriments nécessaires à la maturation de ses œufs. Très sensible aux mouvements, Aedes peut piquer plusieurs hôtes pour un même repas sanguin. Des épidémies de dengue peuvent donc survenir même avec de faibles indices vectoriels, ce qui nécessite une surveillance régulière et spatialement représentative des territoires à risque vectoriel (Fontenille et al., 2009).

L'œuf peut résister près de deux années en milieu sec et mettra en moyenne 3 jours pour éclore une fois submergé. Les stades larvaires et nymphaux sont aquatiques, ils durent en tout de 7 à 17 jours selon les conditions de température et de disponibilité en nourriture avant de produire un adulte, aérien, dont la durée de vie peut s'établir autour de 25 jours (Christophers, 1960).

L'écologie du moustique permet son contrôle. Privilégiant les milieux humides et sombres pour se reposer, on le trouve dans les maisons, principalement dans les salles d'eau (toilettes, salle de bain, cuisine). À la recherche de gîtes de ponte en eau, de préférence claire, les indices larvaires sont élevés dans un périmètre restreint autour des maisons (Harrington et al., 2005). Ces relations d'influence de l'environnement sur le moustique motivent alors les stratégies de gestion de l'environnement: destructions ciblées en milieux domiciliaires (Tun-Lin et al., 2009), fumigation des espaces publics (OMS, 2012a) ou encore aménagements des espaces urbains (Gubler, 2011).

\section{L'hôte : l'homme}

La période d'incubation du virus chez l'homme dure entre 4 et 10 jours, suivie d'une possible période de fébrilité de 2 à 7 jours. Après l'incubation du virus, la période de contagiosité dure de 4 à 9 jours (OMS, 2012b). Chez l'homme, le virus reste présent pendant seulement quelques semaines avant d'être éliminé. N'importe lequel des quatre sérotypes (désignés DENV-1, DENV-2, DENV-3 et DENV-4) peut causer une infection virale aiguë caractérisée par une fièvre (DF) ; une éruption cutanée; des maux de tête, des muscles; des douleurs articulaires et des nausées. Dans certains cas, DF peut progresser vers une dengue hémorragique (DHF), forme potentiellement mortelle associée à une fuite plasmatique, des hémorragies gastro-intestinales qui précèdent très souvent l'apparition du syndrome de choc (DSS). La réponse des anticorps à une première infection par un sérotype de la dengue semble fournir une protection croisée aux quatre sérotypes, probablement pour un an ou plus (Salje et al., 2012). Après ce délai, la concentration d'anticorps neutralisant décroît et rend à nouveau l'individu susceptible aux autres sérotypes, mais une immunité complète semble acquise à l'égard du primo sérotype. Un individu peut donc potentiellement développer quatre infections à la dengue. Entre 50 et $80 \%$ des infections à la dengue sont cependant asymptomatiques (Grange et al., 2014). Ce différentiel de vulnérabilité individuelle dépend de nombreux facteurs tels que la génétique, l'immunité ou encore l'âge (Huy et al., 2013).

Chez l'hôte, les effets de contextes sont importants dans l'évaluation du risque de contamination. La connaissance des risques de contamination, des mesures de protection, les pratiques de gestion de son environnement immédiat et le mode de vie de l'individu sont des facteurs associés à ce risque (Setbon et Raude, 2009). On souligne également l'importance du contexte sociospatial de la vulnérabilité, notamment par les lieux que les individus fréquentent. Si le moustique est le vecteur du virus dans un espace de vie limité à une centaine de mètres du lieu de son émergence (Ordóñez-Gonzalez et al., 2001), il laisse à l'homme le rôle du porteur sur de plus longues distances : aux échelles urbaine (Stoddard et al., 2013), nationale (Cummings et al., 2004) ou mondiale (Tatem et. al, 2006).

La plupart des systèmes de surveillance et de contrôle intègrent dans leur protocole le suivi des cas de dengue. Ceux-ci sont enregistrés auprès des structures de soins et peuvent permettre d'enclencher des actions ciblées pour limiter l'expansion de la maladie. Ainsi à Delhi (Inde), les actions de fumigation sont en partie basées sur la localisation des cas de dengue recensés par le système de surveillance des hôpitaux sentinelles (Telle, 2011). Cependant, compte tenu d'une part de la forte proportion de cas asymptomatiques à la dengue et d'autre part de la sous-déclaration des cas, la dengue n'étant pas une maladie à déclaration obligatoire dans de nombreux pays, la seule surveillance des cas de dengue ne permet pas d'assurer un suivi efficace de l'extension possible du virus (Daudé et Vaguet, 2015).

\section{Le virus : DENV 1-2-3-4}

Le virus de la dengue appartient à la famille Flaviviridae, qui comprend entre autres les virus de la Fièvre Jaune, de l'Encéphalite Japonaise et de la Vallée du Nil occidental (Weaver et Vasilakis, 2009). Chez le moustique, le virus pénètre la paroi stomacale et se réplique jusqu'aux glandes salivaires. Ce processus dure de une à deux semaines, selon les températures. Si l'impact du virus chez le moustique est peu renseigné, il reste présent dans le moustique durant toute sa vie, ce dernier est donc contaminant jusqu'à sa mort. Et si les femelles Ae. aegypti infectées par la dengue meurent, en moyenne, deux fois plus rapidement que les femelles saines (Maciel-de-Freitas et al., 2011), cela n'est pas suffisant pour éviter une série de piqûres contaminantes.

La capacité du virus à évoluer et devenir plus infectieux pour Ae. albopictus ou même pour d'autres espèces 
de moustiques reste un champ de recherche important (Jaenisch et al., 2013). Le virus ne survit pas dans l'environnement, mais il peut être transmis aux œufs d'un moustique infecté, phénomène dit de transmission verticale, et ainsi survivre à des périodes météorologiques défavorables (Joshi et al., 2002).

\section{L'environnement}

Le poids des facteurs environnementaux qui ressortent de l'épidémiologie de la dengue dépendent du niveau de granularité spatiotemporel considéré (Eisenberg et al., 2007). Les changements d'échelles, du micro au macroscopique, éloignent progressivement de la recherche de causes directes favorables au cycle de contamination, les densités vectorielles par exemple, vers la recherche de facteurs indirects qui agissent sur ces causes, par des liens de plus en plus distants, telles que les variations climatiques. L'estimation des populations à risque de la dengue à l'échelle du monde s'appuie ainsi sur des données climatiques, des taux d'urbanisation ou encore des indicateurs de pauvreté (Bhatt et al., 2013). Cependant si ces facteurs influencent potentiellement le cycle de contamination, ils ne sont pas systématiquement identifiés comme tels, car le résultat des causalités non linéaires entre ces facteurs dépend des contextes locaux. La connaissance de ces relations permet malgré tout la mise en œuvre d'actions à différentes échelles pour tenter d'améliorer les conditions de santé publique : contrôle régulier des gîtes en eau (Abe et al., 2005), mesures de protection intra domiciliaires (Singh et al., 2011), amélioration des conditions d'accès à l'eau des populations (Schmidt et al., 2011), interventions selon les types d'usages du sol (Thammapalo et al., 2008) ou encore politiques de planification urbaine (De Mattos Almeida et al., 2007).

Cette variabilité des facteurs selon les échelles spatiales se retrouve au niveau temporel : les températures journalières influencent les pics d'agressivité du moustique et la probabilité de transmission du virus au vecteur (Lambrechts et al., 2011), ainsi que la période d'incubation extrinsèque (Watts et al., 1987), alors que leurs variations annuelles introduisent des cycles dans l'évolution du risque vectoriel (Yang et al., 2009). Une variable environnementale peut donc être pertinente à différents niveaux d'observations, moyennant un calibrage de son emprise spatiale et temporelle aux processus décrits.

\section{... et leurs interactions}

La stratégie de contrôle vectoriel mise en place par les autorités de Singapour (Ooi et al., 2006) a permis de diminuer la population de moustiques et de contrôler les épidémies de dengue pendant de nombreuses années. Ce qui a progressivement conduit à une baisse de l'immunité globale de la population. L'introduction d'une nouvelle souche a alors provoqué de nouvelles épidémies de dengue après plus de quinze années de faible incidence. Ce cas illustre la difficulté de pouvoir anticiper sur les évolutions de la dengue en se basant sur une observation statique de son système ou sur un seul de ses composants, au détriment de la surveillance et de l'action sur les autres. Le schéma du système de la dengue proposé dans la figure 1 représente les différentes interactions entre composants ainsi que les principaux facteurs qui leur sont associés, à différentes échelles, et qui peuvent agir sur sa dynamique globale.

Les dynamiques possibles du système de la dengue dépendent donc à la fois de l'état de ses composants à un moment donné et de leurs interactions (Fig. 1). Ces états, ou attributs des composants, sont relatifs aux échelles géographiques et temporelles sélectionnées. La recherche de proxys est en général motivée par la volonté de réduire la quantité d'information qui est nécessaire pour estimer les conditions initiales du système : l'évaluation de la densité de gîtes potentiels à l'échelle d'un pays n'est pas nécessaire pour estimer la population vectorielle, les grandes variables climatiques peuvent suffire (Jetten et Focks, 1997). En revanche, à un niveau très fin, celui du bâtiment par exemple, la présence de gîtes en eau pour la ponte du moustique et les densités d'hôtes sont, en plus des températures, des déterminants importants de la survie du moustique et donc du risque de transmission en cas de présence du virus. Si l'objectif est d'explorer le risque de dengue à des échelles de temps et d'espace relativement fines, il devient alors nécessaire d'augmenter le nombre de critères à observer pour étudier précisément le poids relatif de ces facteurs et de leurs interactions.

Si la connaissance de l'état initial du système est un élément important pour évaluer le risque de dengue, elle n'est pas suffisante car il évolue en permanence, du fait des dynamiques internes des composants et de leurs effets sur les autres composants, au travers des interactions. Ces interactions sont le médium de la dynamique globale du système dans la mesure où elles génèrent des boucles de rétroactions non linéaires (Tab. 1). Une boucle de rétroaction positive existe potentiellement entre la population d'hôtes et celle de moustiques : l'augmentation du nombre d'hommes contaminés s'accompagne potentiellement d'une augmentation du nombre de moustiques contaminés. Une boucle de rétroaction négative s'opère entre le taux d'immunité de la population d'hôtes et la prévalence du virus : la hausse du taux d'immunité à un sérotype freine la progression de la population virale de ce sérotype, pas seulement en réduisant le nombre d'individus toujours susceptibles, mais aussi via l'effet d'immunité de groupe. Les individus 


\begin{tabular}{|c|c|c|}
\hline 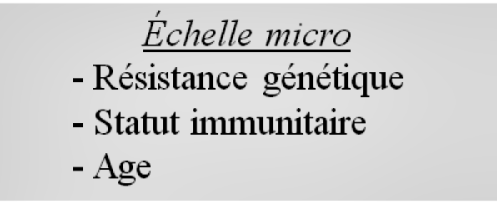 & $\begin{array}{l}\quad \underline{E c h e l l e ~ m e ́ s o ~} \\
\text { - Prévalence d'anticorps } \\
\text { - Infection asymptomatique } \\
\text { - Culture du risque }\end{array}$ & $\begin{array}{l}\quad \text { Échelle macro } \\
\text { - Densité de population } \\
\text { - Mobilité } \\
\text { - Niveau de richesse }\end{array}$ \\
\hline $\begin{array}{l}\text { Echelle micro } \\
\text { - Virulence } \\
\text { - Génétique } \\
\text { - Quasi-espèce } \\
\text { Echelle méso } \\
\text { - Sérotype } \\
\text { - Clade } \\
\text { - Antériorité locale } \\
\text { Echelle macro } \\
\text { - Origine régionale } \\
\text { - Compétition entre } \\
\text { sérotypes }\end{array}$ & 12 & $\begin{array}{l}\text { Echelle micro } \\
\text { - État gravide } \\
\text { - Age } \\
\text { - Comportement de } \\
\text { prédation } \\
\text { Echelle méso } \\
\text { - Taux de piqure } \\
\text { - Taux de survie } \\
\text { - Aire de déplacement } \\
\text { Échelle macro } \\
\text { - Densité } \\
\text { - Taux de contamination } \\
\text { - Pyramide des âges }\end{array}$ \\
\hline $\begin{array}{l}\frac{E \text { Echelle micro }}{\text { - Container en eau }} \\
\text { - Présence de moustiquaires } \\
\text { - Taux d'humidité }\end{array}$ & $\begin{array}{l}\frac{\text { Echelle méso }}{\text { - Gestion de l'eau et des déchets }} \\
\text { - Modes d'occupation du sol } \\
\text { - Densité de l'habitat }\end{array}$ & $\begin{array}{l}\quad \frac{E \text { Echelle macro }}{\text { - Température / Précipitation }} \\
\text { - Planification urbaine } \\
\text { - Interactions spatiales }\end{array}$ \\
\hline
\end{tabular}

Fig. 1. Le système de la dengue, ses composants et leurs interactions.

Pour chacun des composants hôte/vecteur/virus/environnement sont mentionnés les principaux facteurs de la dynamique du composant ou du système, à différentes échelles.

toujours susceptibles sont ainsi moins exposés grâce à l'état d'immunité des individus qui les entourent (Anderson et May, 1991).

Dynamiques multiniveaux, rétroactions non linéaires, fortes sensibilités aux conditions initiales et émergences sont donc les marqueurs de la complexité de la dengue qui expriment la grande variabilité géographique de la contribution des facteurs dans l'expression de la maladie.

Dans sa stratégie globale pour la prévention et le contrôle de la dengue, l'OMS (2012a) souligne le rôle que pourraient jouer les bases de données géographiques et les modèles spatiotemporels dans l'identification des risques épidémiques à différentes échelles, et notamment aux échelles micro-locales des espaces urbains.

\section{Diagnostics territoriaux et modélisations spatiotemporelles de la dengue}

Les études rétrospectives, le suivi épidémiologique et entomologique ne constituent encore que très rarement des ressources pour la prévention et la gestion des épidémies de dengue (OMS, 2012). Or ces dimensions sont porteuses de sens. Ainsi l'épidémie de dengue de
2010 à Delhi a coïncidé avec l'organisation des jeux du Commonwealth. Ceux-ci ont non seulement engendré le passage de milliers de migrants durant plusieurs semaines, introduisant potentiellement des virus ainsi que des individus susceptibles, mais ils ont aussi nécessité d'importants travaux d'infrastructures, ce qui a entraîné une augmentation de la population vectorielle (Sharma et al., 2012). Estimer l'impact de ces changements ne peut se faire qu'à partir d'une information territoriale régulièrement mise à jour. Les bases de données doivent être à la fois entomologiques et épidémiologiques, mais aussi intégrer les dimensions sociodémographiques et géographiques pour couvrir tout le système de la dengue (Fig. 2).

\section{Les SIG pour la surveillance des territoires à risques de dengue}

Les systèmes d'informations géographiques (SIG) sont des systèmes de gestion de bases de données localisées et thématiques, utiles pour les systèmes de surveillance de la dengue grâce à la géolocalisation des cas et des indices vectoriels (Eisen et Lozano-Fuentes, 2009). L'utilisation d'outils multimédias équipés de fonction GPS sur le terrain, pour le passage de questionnaires, la 


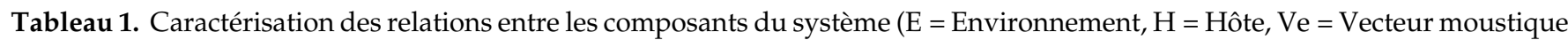
et $\mathrm{Vi}=$ Virus).

\begin{tabular}{|c|c|c|c|c|}
\hline Relation & De type & Porte sur & Surveillance & Contrôle \\
\hline $\begin{array}{c}1 \\
(c f . \text { Fig. } 1) \\
\mathrm{E} \rightarrow \mathrm{Ve}\end{array}$ & Favorise/contraint & $\begin{array}{l}\text { Densité de gîtes ; } \\
\mathrm{T}^{\circ} \text { et } \mathrm{P}^{\circ}\end{array}$ & $\begin{array}{l}\text { Dénombrement des gîtes potentiels } \\
\text { par unité d'espace }\end{array}$ & $\begin{array}{l}\text { Vidange régulière des gîtes en eau ; } \\
\text { contrôles vectoriels durant les } \\
\text { périodes de confort du moustique }\end{array}$ \\
\hline$\stackrel{2}{\mathrm{Ve}} \rightarrow \mathrm{E}$ & $\begin{array}{l}\text { Attiré par ; } \\
\text { Localisation }\end{array}$ & Lieu humide & $\begin{array}{l}\text { Container index ; House Index ; } \\
\text { Bréteau Index }\end{array}$ & $\begin{array}{l}\text { Ovitrap, fumigation, moustiquaires } \\
\text { de fenêtre }\end{array}$ \\
\hline $\mathrm{H} \rightarrow \mathrm{Ve}$ & Transmet & Sang ; virus & $\begin{array}{l}\text { Système de surveillance et de } \\
\text { déclaration des cas de dengue }\end{array}$ & $\begin{array}{l}\text { Destruction des moustiques dans } \\
\text { le domicile du malade }\end{array}$ \\
\hline $\mathrm{Ve} \stackrel{4}{\rightarrow} \mathrm{H}$ & Transmet & Virus & Taux de piqûres contaminantes & $\begin{array}{l}\text { Mesure individuelle de protection } \\
\text { contre les piqûres }\end{array}$ \\
\hline$\stackrel{5}{H \rightarrow E}$ & Aménage & $\begin{array}{l}\text { Occupation } \\
\text { du sol }\end{array}$ & $\begin{array}{l}\text { Repérage des chantiers } \\
\text { de construction en zone urbaine }\end{array}$ & $\begin{array}{l}\text { Législation particulière sur ces } \\
\text { zones }\end{array}$ \\
\hline$\stackrel{6}{\mathrm{E} \rightarrow \mathrm{H}}$ & Contraint & $\begin{array}{l}\text { Réseaux } \\
\text { de circulation }\end{array}$ & $\begin{array}{l}\text { Connaissance des flux de population } \\
\text { et de concentration }\end{array}$ & $\begin{array}{l}\text { Contrôle vectoriel sur les principaux } \\
\text { lieux d'échanges de population }\end{array}$ \\
\hline$\stackrel{7}{\mathrm{Vi} \rightarrow \mathrm{H}}$ & Infecte & Cellules & Taux de plaquettes dans le sang & $\begin{array}{l}\text { Transfusion de globules rouges } \\
\text { et de plaquettes }\end{array}$ \\
\hline$\stackrel{8}{\mathrm{H} \rightarrow \mathrm{Vi}}$ & Réplique/lutte & Anticorps & $\begin{array}{l}\text { Séroprévalence au sein de la } \\
\text { population }\end{array}$ & Mise au point d'un vaccin \\
\hline$\stackrel{9}{\mathrm{Vi} \rightarrow \mathrm{Ve}}$ & Pénètre & Cellules & Taux de moustiques contaminés & $\begin{array}{l}\text { Moustique transgénique non por- } \\
\text { teur de la dengue }\end{array}$ \\
\hline $\begin{array}{l}10 \\
\mathrm{Ve} \rightarrow \mathrm{Vi}\end{array}$ & Réplique & Virus & $\begin{array}{l}\text { Dissémination dans le corps } \\
\text { du moustique }\end{array}$ & $\begin{array}{l}\text { Lâché de mâles stériles pour éviter } \\
\text { la transmission aux œufs ; réduire } \\
\text { durée de vie (par ex. lutte biologique) }\end{array}$ \\
\hline $\mathrm{E} \rightarrow \mathrm{Vi}$ & Influence & Température $\left(\mathrm{T}^{\circ}\right)$ & $\begin{array}{l}\text { Vitesse de développement } \\
\text { extrinsèque }\end{array}$ & $\begin{array}{l}\text { Réduire la durée de vie du } \\
\text { moustique }\end{array}$ \\
\hline $\begin{array}{c}12 \\
\mathrm{Vi} \rightarrow \mathrm{E}\end{array}$ & Localisation & Présence de virus & $\begin{array}{l}\text { Présence de virus dans } \\
\text { l'environnement }\end{array}$ & $\begin{array}{l}\text { Aucun a priori, le virus ne persiste } \\
\text { pas longtemps dans la nature }\end{array}$ \\
\hline
\end{tabular}

Les colonnes indiquent respectivement le sens de la relation, sa nature, l'objet sur lequel elle est agissante, les moyens de son observation et de son contrôle.

saisie d'informations et de photos, permet d'optimiser les échanges de format et la centralisation des données (Lwin et al., 2013). Lorsqu'ils existent, ces systèmes permettent de suivre les foyers d'endémie, les populations vectorielles (Palaniyandi, 2014) et d'interagir avec les acteurs de la prévention et de la lutte ${ }^{1}$. Ces données,

\footnotetext{
1 Voir à ce titre le système mis en place à Singapour : www.dengue.gov.sg/.
}

comparées avec des données socioéconomiques et environnementales, permettent alors la mise en œuvre de méthodes d'épidémiologie spatiale (Getis, 1999) pour la réalisation de diagnostics territoriaux. L'identification de disparités des risques selon différents découpages socioenvironnementaux et les analyses rétrospectives sur les épidémies en sont les principaux atouts.

L'étude des regroupements spatiotemporels des cas de dengue a ainsi permis d'estimer le poids de la 


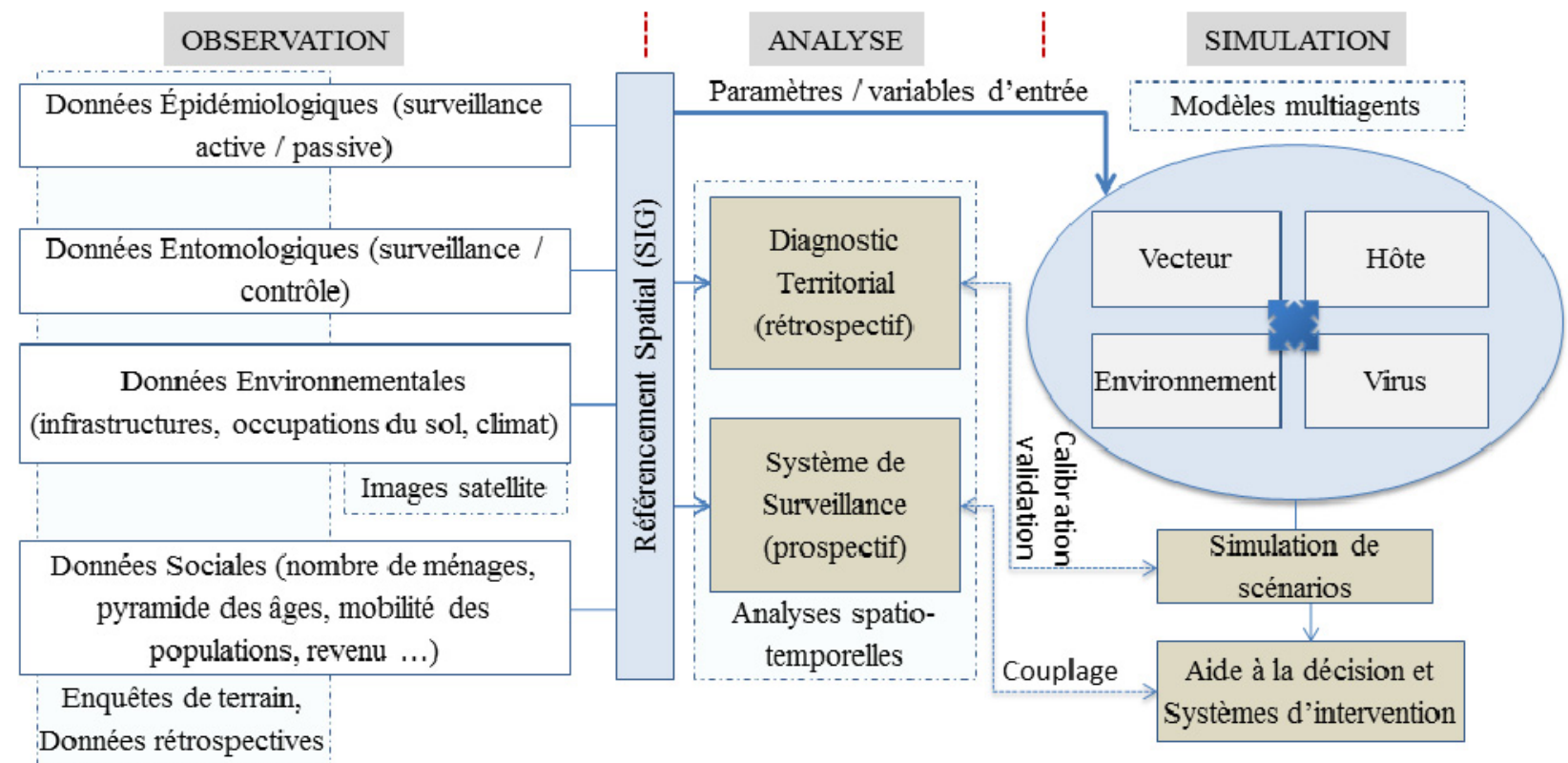

Fig. 2. Cadre général d'une démarche intégrée pour l'observation, l'analyse et la modélisation du système complexe de la dengue.

contamination locale et de rechercher l'échelle pertinente pour le contrôle vectoriel (Kan et al., 2008). Les zones de concentration des indices vectoriels en milieu urbain ont pu être calculées à partir de facteurs environnementaux tels que la densité de maisons en briques ou l'absence de réseau de collecte des ordures en Thaillande (Thammapalo et al., 2008). Les corrélations entre des données sociodémographiques (éducation, revenu, taille du ménage) et des données épidémiologiques ont permis d'identifier les populations les plus vulnérables au Brésil (De Mattos Almeida et al., 2007). L'intégration d'images satellites dans la chaîne de traitement permet d'identifier et de caractériser de manière automatique et à petite échelle les zones à risques vectoriels (Fuller et al., 2010). Il devient alors possible d'évaluer et de localiser les risques de dengue et donc d'orienter sur le terrain les campagnes de contrôles vectoriels et la prévention (Rotela et al., 2007). Néanmoins, on ne peut que regretter que ces outils soient pour l'heure largement sousutilisés, en raison de la disparité des données et du manque de formation des personnels de santé publique.

Au-delà des diagnostics territoriaux du système de la dengue, sa modélisation permet d'explorer ses possibles évolutions. Il ne s'agit pas ici de vouloir reproduire quantitativement les chaînes de causalité des épidémies passées, mais de mieux comprendre le fonctionnement qualitatif du système, pour agir sur lui de manière plus harmonieuse. On recherche alors les boucles de rétroactions afin de repérer leurs points critiques, qui conduisent à des changements quantitatifs. Ceci passe par la modélisation et la simulation informatique.

\section{La simulation pour la prospective territoriale des risques de dengue}

La modélisation et la simulation informatique pour explorer les dynamiques de systèmes géographiques et complexes combinent différentes méthodologies, dont les SIG et les modèles à base d'agents (Langlois, 2010). Ces derniers sont définis par des variables et des comportements distribués qui permettent de reproduire la diversité du réel. La simulation permet l'interaction chez les agents, entre eux et avec leur environnement, et sous différentes conditions. Quelles que soient leurs fonctions épistémiques, ces modèles sont utilisés comme des laboratoires virtuels où processus et jeux de données permettent de simuler des scénarios d'évolutions d'une géographie artificielle des phénomènes du vivant.

Prenons le cas de la modélisation du vecteur de la dengue. Dans ce modèle (Maneerat et Daudé, 2015), chaque agent est défini par son genre, son âge et son statut au regard de l'infection. Ses comportements reproduisent les observations et les processus décrits par les entomologistes : méthodes de déplacement et de recherche de repas sanguin, de gîtes de ponte ou de repos sont programmées pour représenter au mieux les aptitudes et les activités du moustique. Multipliés par centaines ou milliers, ils constituent des cohortes de moustiques artificiels sur lesquels le chercheur va expérimenter. Ces agents-moustiques évoluent dans des environnements très hétérogènes, définis selon les besoins et les contraintes du vecteur (Hartemink et al., 2014). De tels agents sont parfaitement adaptatifs à leur milieu; leurs 
capacités de perception et d'action leur permettent d'agir en fonction des contraintes et des opportunités qui se présentent.

Parce que c'est un programme informatique, identifié et unique, il est possible d'enregistrer toutes les actions et l'ensemble des changements d'état qui se produisent dans la vie d'un agent. Combien d'hôtes un moustique infecté va-t-il contaminer ? Où ont lieu les contaminations? Quelles sont les zones privilégiées de ponte? Quelles sont les aires de dispersion des moustiques dans différents contextes urbains? En apportant des réponses à ce type de question, la simulation permet d'expérimenter diverses stratégies de contrôle vectoriel (Almeida et al., 2010).

Comme on l'a vu dans la figure 2, l'intérêt est de faire coexister et interagir des agents structurellement différents, ici les quatre entités du système de la dengue. L'hétérogénéité environnementale se modélise via une classe d'agents définis par des attributs : nombre de gîtes potentiels, densité de bâti ou d'espace vert par exemple. Ces agents spatiaux intègrent des processus qui permettent de simuler l'évolution de leurs variables d'état, comme la mise en eau des gîtes potentiels selon les conditions météorologiques. Enfin, l'autre entité biologique du système, l'hôte, est modélisée pour se déplacer dans cet environnement alors que l'entité virus peut s'inclure dans l'un ou l'autre des agents hôte et/ou vecteur, pour produire un modèle de contamination/infection.

La structure d'un métamodèle de simulation - nombre de classes d'agents, de paramètres, de comportements, $\mathrm{d}$ 'interactions - soulève la question de sa vérification et de sa calibration (Treuil et al., 2008). La vérification du code informatique permet de s'assurer que celui-ci réalise ce qui est prévu dans l'ontologie du modèle. Sa calibration consiste à vérifier que le modèle produit, dans certaines conditions paramétriques connues, des dynamiques observées dans le monde réel. La distribution des paramètres et des variables dans le modèle doit donc intégrer les dernières connaissances aussi bien des points de vue géographique, entomologique, biologique que social, ce qui justifie un travail pluridisciplinaire (Magori et al., 2009). À partir du modèle spatialisé, comportemental et distribué sur des millions d'agents, des plans d'expériences permettent d'étudier de manière systématique le comportement du modèle (Ellis et al., 2011). L'étude de la sensibilité du modèle aux variations de paramètres permet de rechercher des valeurs seuils à partir desquelles le système global, ou un de ses soussystèmes, connaît un changement dans sa dynamique par rapport au système étudié. La possibilité de répliquer la même expérience permet de produire des portraits statistiques robustes et comparables aux enquêtes épidémiologiques ou entomologiques. Cela permet également de produire des données statistiques difficilement observables dans la réalité, comme les lieux de transmission privilégiés du virus entre hôtes aux vecteurs, donnant ainsi de nouvelles pistes de recherches pour la lutte contre la dengue.

L'une des promesses d'une modélisation comportementale (moustique, virus, hôte) pour les maladies à transmission vectorielle repose sur le postulat que, quel que soit le lieu, les comportements de prédation du vecteur ou de mobilité de l'hôte sont similaires, seules changent les données. Un modèle comportemental du vecteur est donc a priori structurellement transférable. En changeant les conditions environnementales et sociales du modèle, les chercheurs disposent alors d'un laboratoire artificiel pour réaliser des expériences : quel est le rôle de la distribution spatiale des gîtes potentiels, et le différentiel de leur densité, sur l'abondance des populations vectorielles? Quels sont les effets d'une réduction ciblée de ces gîtes selon leur type (remplissage manuel versus climatique) et leur localisation (lieu public versus lieu privé) sur la densité de moustiques ? Les capacités d'adaptation spatiale du vecteur annulent-elles les effets attendus de ces stratégies (Beebe $e t$ al., 2009), et dans quels délais ? Le confinement des personnes infectées et symptomatiques ainsi que la destruction ciblée des moustiques adultes dans le voisinage des cas index sontils un moyen efficace pour freiner la progression de l'épidémie ? Quels sont le rôle et le poids de la mobilité quotidienne des hommes dans la diffusion du virus (Mpolya et al., 2014) ?

Malgré l'intérêt de ce type de modélisation pour l'étude de la dengue, peu de travaux ont pour l'instant été développés (Tab. 2). Si les aspects purement techniques peuvent être écartés pour expliquer ce relatif désintérêt ${ }^{2}$, il faut mettre en avant la difficulté de paramétrer ce type de modèles, qui peut être un frein à leurs développements, ainsi que les difficultés de valider les résultats selon les méthodes de comparaison « traditionnelles » entre l'observé et le simulé. Il est également probable que le temps de développement d'un modèle de simulation, parfois plus long que le temps contractualisé d'un projet de recherche, explique ce nombre restreint de publications.

\section{Conclusion}

L'émergence ou la réémergence de maladies transmises par des insectes vecteurs est une préoccupation majeure de santé publique. En attente de vaccin et face à la prolifération d'un vecteur compétent, la dengue

\footnotetext{
2 De nombreuses plateformes libres de droit proposent aux chercheurs des langages de manipulation des connaissances qui ne nécessitent pas une compétence spécifique en programmation informatique, ce qui favorise le travail pluridisciplinaire.
} 
Tableau 2. Exemples de modèles à base d'agents spatialisés traitant au moins de l'un des composants du système de la dengue ( $\mathrm{E}=$ environnement, $\mathrm{H}=$ hôte, $\mathrm{Ve}=$ vecteur moustique et $\mathrm{Vi}=$ virus $)$.

\begin{tabular}{|c|c|c|c|c|}
\hline Objectif & $\begin{array}{l}\text { Composants } \\
\text { modélisés }\end{array}$ & Sous-modèles & Principaux résultats & Référence \\
\hline \multirow{2}{*}{$\begin{array}{l}\text { Simuler les dynamiques de population } \\
\text { d'Ae. aegypti à micro-échelle (quelques } \\
\text { maisons) et disposer d'un modèle bio- } \\
\text { logique détaillé pour tester l'efficacité } \\
\text { de scénarios de contrôle vectoriels }\end{array}$} & E : container \& maison & $\begin{array}{l}\text { Transition de stocks } \\
\text { entre les stades } \\
\text { aquatiques }\end{array}$ & \multirow{2}{*}{$\begin{array}{l}\text { Le déplacement des vec- } \\
\text { teurs entre les } \\
\text { maisons réduit les effets } \\
\text { attendus de l'hétérogé- } \\
\text { néité des maisons en } \\
\text { termes de présence } \\
\text { de containers sur la } \\
\text { densité de vecteurs }\end{array}$} & \multirow[b]{2}{*}{$\begin{array}{l}\text { Magori } \\
\text { et al., } 2009\end{array}$} \\
\hline & $\begin{array}{l}\text { Ve : stocks des stades } \\
\text { aquatiques, } \\
\text { agent(ag.)-adulte }\end{array}$ & $\begin{array}{l}\text { Déplacement entre } \\
\text { les maisons des } \\
\text { ag.-adultes }\end{array}$ & & \\
\hline \multirow{3}{*}{$\begin{array}{l}\text { Présenter un modèle de simulation des } \\
\text { dynamiques de population } \\
\text { d'Ae. Aegypti. Disposer d'un modèle } \\
\text { biologique réaliste pour tester les effets } \\
\text { de lâchés de mâles stériles sur ces } \\
\text { dynamiques }\end{array}$} & E : grille d'ag. cellules & $\begin{array}{l}\text { Fluctuation des niveaux } \\
\text { d'humidité par cellule }\end{array}$ & \multirow{3}{*}{$\begin{array}{l}\text { Pas de résultat } \\
\text { significatif, } \\
\text { présentation du modèle }\end{array}$} & \multirow{3}{*}{$\begin{array}{l}\text { Isidoro } \\
\text { et al., } 2009\end{array}$} \\
\hline & $\begin{array}{l}\text { Ve : ag.-femelle, } \\
\text { ag.-mâle \& } \\
\text { ag.-mâle-stérile }\end{array}$ & $\begin{array}{l}\text { Se reproduire, prendre } \\
\text { un repas sanguin, } \\
\text { pondre, croître }\end{array}$ & & \\
\hline & H : ag.-humain & $\begin{array}{l}\text { Déplacement aléatoire } \\
\text { dans la grille } \\
\end{array}$ & & \\
\hline \multirow{3}{*}{$\begin{array}{l}\text { Étudier les effets de la variation dans } \\
\text { l'espace intra et péri-domiciliaire du } \\
\text { nombre de pièges à moustique sur la } \\
\text { densité de vecteurs }\end{array}$} & $\begin{array}{l}\text { E : ag.-container, } \\
\text { ag.-végétation, } \\
\text { ag.-ovitrap }\end{array}$ & $\begin{array}{l}\text { Fluctuation du volume } \\
\text { d'eau et de l'émission } \\
\text { de traces. L'ag.-ovitrap } \\
\text { détruit les œufs }\end{array}$ & \multirow{3}{*}{$\begin{array}{l}\text { Résultats simulés } \\
\text { comparés avec une expé- } \\
\text { rience, meilleure effica- } \\
\text { cité des résultats lorsque } \\
\text { les ovitraps sont placés à } \\
\text { l'extérieur des maisons }\end{array}$} & \multirow{3}{*}{$\begin{array}{l}\text { Almeida } \\
\text { et al., } 2010\end{array}$} \\
\hline & $\begin{array}{l}\text { Ve : ag.-femelle, } \\
\text { ag.-mâle }\end{array}$ & \begin{tabular}{|l|} 
Transitions entre états \\
pré-adultes, se dépla- \\
cer, chercher un lieu de \\
repos, un repas sanguin \\
ou sucré, se reproduire, \\
pondre
\end{tabular} & & \\
\hline & $\begin{array}{l}\text { H : ag.-humain, } \\
\text { ag.-chien, ag.-chat }\end{array}$ & $\begin{array}{l}\text { Se déplacer selon un } \\
\text { programme d'activité }\end{array}$ & & \\
\hline \multirow[t]{2}{*}{$\begin{array}{l}\text { Étudier le comportement de prédation } \\
\text { du moustique et sa distribution } \\
\text { spatiale }\end{array}$} & $\begin{array}{l}\text { E : grille } \\
\text { d'ag.-cellules }\end{array}$ & $\begin{array}{l}\text { Emission et diffusion } \\
\text { de traces, modèle de } \\
\text { simulation du vent }\end{array}$ & \multirow[t]{2}{*}{$\begin{array}{l}\text { Les taux de contacts hôte- } \\
\text { vecteur augmentent avec } \\
\text { la dispersion des hôtes } \\
\text { dans une zone }\end{array}$} & \multirow[t]{2}{*}{$\begin{array}{l}\text { Cummins } \\
\text { et al., } 2012\end{array}$} \\
\hline & Ve : ag.-moustique & Suivre une trace $\left(\mathrm{Co}_{2}\right)$ & & \\
\hline \multirow{3}{*}{$\begin{array}{l}\text { Simuler les dynamiques spatiales des } \\
\text { quatre souches de la dengue }\end{array}$} & $\begin{array}{l}\text { E : grille } \\
\text { d'ag.-cellules }\end{array}$ & Aucun & \multirow{3}{*}{$\begin{array}{l}\text { Un processus stochas- } \\
\text { tique de transmission et } \\
\text { un arrangement spatial } \\
\text { des hôtes peuvent expli- } \\
\text { quer la présence de plu- } \\
\text { sieurs épidémies } \\
\text { annuelles }\end{array}$} & \multirow{3}{*}{$\begin{array}{l}\text { Lourenço } \\
\text { et Recker, } \\
2013\end{array}$} \\
\hline & H : ag.-humain & $\begin{array}{l}\text { Déplacement, incuba- } \\
\text { tion intrinsèque }\end{array}$ & & \\
\hline & Ve : ag.-moustique & Incubation extrinsèque & & \\
\hline \multirow{3}{*}{$\begin{array}{l}\text { Simuler les dynamiques de popula- } \\
\text { tions d'Ae. aegypti à méso-échelle } \\
\text { (un quartier) à partir d'un modèle } \\
\text { comportemental détaillé }\end{array}$} & $\begin{array}{l}\text { E : ag. de type maillage } \\
\text { irrégulier }\end{array}$ & $\begin{array}{l}\text { Modèle météorologique } \\
\text { selon le type d'utilisa- } \\
\text { tion du sol. Les stocks } \\
\text { de moustiques imma- } \\
\text { tures sont gérés par E. }\end{array}$ & \multirow{3}{*}{$\begin{array}{l}\text { Validation du modèle sur } \\
\text { la dispersion de cohortes } \\
\text { de moustiques selon dif- } \\
\text { férentes configurations } \\
\text { spatiales ; reproduction } \\
\text { de la pyramide des âges } \\
\text { et âge limite moyen du } \\
\text { vecteur }\end{array}$} & \multirow{3}{*}{$\begin{array}{l}\text { Maneerat } \\
\text { et Daudé, } \\
2015\end{array}$} \\
\hline & H : stock dans E & $\begin{array}{l}\text { Mobilité quotidienne } \\
\text { des hôtes }\end{array}$ & & \\
\hline & Ve : ag.-moustique & $\begin{array}{l}\text { Se déplacer, rechercher } \\
\text { une cible, piquer, pondre }\end{array}$ & & \\
\hline
\end{tabular}


semble désormais installée pour longtemps au cœur des principales problématiques de santé publique.

Le cadre systémique et complexe de la dengue développé dans cet article donne une vision multiniveaux des composants du système hôte/vecteur/virus/environnement et ouvre de nombreuses pistes de recherche sur le rôle des interactions dans les dynamiques possibles de la maladie. Dans ce contexte, la modélisation à base d'agents spatialement explicite est présentée. Elle permet une modélisation réaliste des comportements des entités élémentaires responsables du cycle de transmission et intègre toute la richesse des bases de données issues des systèmes d'information géographique : hétérogénéité et mobilité des populations humaines, dynamique des densités vectorielles ou encore changements des conditions environnementales conduisent alors à tester de nombreux scénarios d'évolutions du système, à la fois pour explorer les mécanismes passés, mais également anticiper ceux à venir. La dengue en tant que système complexe implique alors un travail pluridisciplinaire où la modélisation à base d'agents spatialisés joue un rôle important dans le dialogue entre les chercheurs.

\section{Remerciements}

Cet article a été écrit dans le cadre des projets suivants: DENFREE (Dengue Research Framework for Resisting Epidemics in Europe, grant agreement: 282 378), septième programme de la Commission européenne (FP7) et AEDESS (Analyse de l'émergence de la dengue et simulation spatiale, 10 CEPL 004-01) de l'Agence nationale de la recherche. Les auteurs remercient leurs partenaires Alexandre Cebeillac, Bertrand Lefebvre, Somsakun Maneeerat, Renaud Misslin, Patrick Taillandier, Yvette Vaguet (Université de Rouen) et Olivier Telle (Institut Pasteur). Les auteurs remercient également les relecteurs pour l'ensemble de leurs suggestions.

\section{Références}

Abe, M., McCall, P., Lenhart, A., Villegas, E., Kroeger, A., 2005. The Buen Pastor cemetery in Trujillo, Venezuela: Measuring dengue vector output from a public area, Tropical Medicine E International Health, 10, 6, 597-603.

Almeida, S.J. de, Ferreira, R., Eiras, Á., Obermayr, R., Geierd, M., 2010. Multi-agent modeling and simulation of an Aedes aegypti mosquito population, Environmental Modelling $\mathcal{E}$ Software, 25, 12, 1490-1507.

Anderson, R.M., May, R.M., 1991. Infectious Diseases of Humans: Dynamics and Control, Oxford, Oxford University Press.

Beebe, N., Cooper, R., Mottram, P., Sweeney, A., 2009. Australia's dengue risk driven by human adaptation to climate change, PLoS Neglected Tropical Diseases, 3, 5, e429.
Bhatt, S., Gething, P., Brady, O., Messina, J., Farlow, A., Moyes, C., Drake, J., Brownstein, J., Hoen, A., Sankoh, O., Myers, M., George, D., Jaenisch, T., Wint, G., Simmons, C., Scott, T., Farrar, J., Hay, S. 2013. The global distribution and burden of dengue, Nature, 496, 504-507, doi: 10.1038/ nature12060.

Christophers, S.R., 1960. Aedes Aegypti (L.): The Yellow Fever Mosquito, Cambridge, Cambridge University Press.

Cummings, D., Irizarry, R., Huang, N., Endy, T., Nisalak, A., Ungchusak, K., Burke, D., 2004. Travelling waves in the occurrence of dengue hemorrhagic fever in Thailand, Nature, 427, 6972, 344-347.

Cummins, B., Cortez, R., Foppa, I.M., Walbeck, J., Hyman, J.M., 2012. A spatial model of mosquito host-seeking behavior, PLoS Computational Biology, 8, 2, e1002500.

Daudé, É., Vaguet, A., 2015. Surveillance, contrôle et épidémies de dengue en Inde : qui a échoué ?, L'Espace Politique, 26, 2, doi: 10.4000/espacepolitique.3485.

De Mattos Almeida, M.C., Caiaffa, W.T., Assunção, R., Proietti, F., 2007. Spatial vulnerability to dengue in a Brazilian urban area during a 7-year surveillance, Journal of Urban Health: Bulletin of the New York Academy of Medicine, 84, 3, 334-345.

Descloux, E., Mangeas, M., Lengaigne, M., Leroy, A., Menkes, C., Tehei, T., Guillaumot, L., Teurlai, M., Gourinat, A.-C., Benzler, J., 2012. Climate-based models for understanding and forecasting dengue epidemics, PLoS Neglected Tropical Diseases, 6, 2, e1470, doi: 10.1371/journal.pntd.0001470.

Deubel, V., Rodhain, F., 1999. Variations climatiques et dengue : impacts directs et indirects, Médecine et Maladies Infectieuses, 29, 5, 289-295.

Eisen, L., Lozano-Fuentes, S., 2009. Use of mapping and spatial and space-time modeling approaches in operational control of Aedes aegypti and dengue, PLoS Neglected Tropical Diseases, 3, 4, e411, doi: 10.1371/journal.pntd.0000411.

Eisenberg, J., Desai, M., Levy, K., Bates, S., Liang, S., Naumoff, K., Scott, J., 2007. Environmental determinants of infectious disease: A framework for tracking causal links and guiding public health research, Environ Health Perspectives, 115, 8, 1216-1223.

Ellis, A., Garcia, A., Focks, D., Morrison, A., Scott, T., 2011. Parameterization and sensitivity analysis of a complex simulation model for mosquito population dynamics, dengue transmission, and their control, The American Journal of Tropical Medicine and Hygiene, 85, 2, 257-264.

Fontenille, D., Lagneau, C., Lecollinet, S., Lefait-Robin, R., Setbon, M., Tirel, B., Yebakima, A., 2009. La Lutte antivectorielle en France, Marseille, IRD Éditions, coll. Expertise collégiale.

Fuller, D., Troyo, A., Calderon-Arguedas, O., Beier, J., 2010. Dengue vector larval habitats in an urban environment of Costa Rica analysed with ASTER and QuickBird imagery, International Journal of Remote Sensing, 31, 1, 3-11.

Getis, A., 1999. Spatial statistics, in Longley, P., Goodchild, M., Maguire, G., Rhind, D. (Ed.), Geographic Information Systems, New York, John Wiley and Sons, 239-251.

Grange, L., Simon-Loriere, E., Sakuntabhai, A., Gresh, L., Paul, R., Harris, E., 2014. Epidemiological risk factors associated with high global frequency of inapparent dengue virus infections, Frontiers in Immunology, 5, 280. 
Gubler, D., 1998. Population growth, urbanization, automobiles and airplanes: The dengue connection, in Greenwood, B., De Cock, K., (Ed.), New and Resurgent Infections: Prediction, Detection and Management of Tomorrow's Epidemics, London, London School of Hygiene and Tropical Medicine, 117-129.

Gubler, D., 2011. Dengue, urbanization and globalization: The unholy Trinity of the 21st century, Tropical Medicine and Health, 39, 4, 3-11.

Gubler, D., Kuno, G., (Ed.), 1997. Dengue and Dengue Hemorrhagic Fever, Wallingford, UK, CAB International.

Guzman, M., Halstead, S., Artsob, H., Buchy, P., Farrar, J., Gubler, D., Hunsperger, E., Margolis, H., Martínez, E., Nathan, M., Pelegrino, J., 2010. Dengue : a continuing global threat, Nature, 8, 12, doi.org/10.1038/nrmicro2460.

Hales, S., de Wet, N., Maindonald, J., Woodward, A., 2002. Potential effect of population and climate changes on global distribution of dengue fever: An empirical model, Lancet, 360, 9336, 830-834.

Handschumacher, P., Hervouet, J.-P., 2004. Des systèmes pathogènes à la santé publique, une nouvelle dimension pour la géographie de la santé tropicale, Autrepart, 29, 4763.

Harrington, L., Scott, T., Lerdthusnee, K., Coleman, R., Costero, A., Clark, G., Jones, J., Kitthawee, S., Kittayapong, P., Sithiprasasna, R., Edman, J., 2005. Dispersal of the dengue vector Aedes aegypti within and between rural communities, American Society of Tropical Medicine and Hygiene, 72, 2, 209-220.

Hartemink, N., Vanwambeke, S., Purse, B., Gilbert, M., Van Dyck, H., 2014. Towards a resource-based habitat approach for spatial modelling of vector-borne disease risks, Biological Reviews, 32.

Huy, N.T., Van Giang, T., Thuy, D.H., Kikuchi, M., Hien, T.T., Zamora, J., Hirayama, K., 2013. Factors associated with dengue shock syndrome: A systematic review and metaanalysis, PLoS Neglected Tropical Diseases, 7, 9, e2412, doi: 10.1371/journal.pntd.0002412.

Isidoro, C., Fachada, N., Barata, F., Rosa, A., 2009. Agent-based model of Aedes aegypti population dynamics, Progress in Artificial Intelligence, Berlin, Springer-Verlag, 53-64.

Jaenisch, T., IDAMS, Sakuntabhai, A., DENFREE, WilderSmith, A., DengueTools, 2013. Dengue research funded by the European Commission-scientific strategies of three European dengue research consortia, PLoS Neglected Tropical Diseases, 7, 12, e2320, doi: 10.1371/journal.pntd. 0002320.

Jetten, T.H., Focks, D.A., 1997. Potential changes in the distribution of dengue transmission under climate warming, The American Journal of Tropical Medicine and Hygiene, 57, 3, 285297.

Joshi, V., Mourya, D., Sharma, R., 2002. Persistence of dengue3 virus through transovarial transmission passage in successive generations of Aedes aegypti mosquitoes, The American Journal of Tropical Medicine and Hygiene, 67, 158161.

Kan, C., Lee, P., Wen, T., Chao, D., Wu, M., Lin, N., Scott Y., Shang, C., Fan, I., Shu, P., Huang, J., King, C., Pai, L., 2008. Two clustering diffusion patterns identified from the 20012003 dengue epidemic, Kaohsiung, Taiwan, The American Journal of Tropical Medicine and Hygiene, 79, 3, 344-352.
Kitron, U., 1998. Landscape ecology and epidemiology of vector-borne diseases: Tools for spatial analysis, Journal of Medical Entomology, 35, 435-445.

Lambrechts, L., Paaijmans, K., Fansiri, T., Carrington, L., Kramer, L., Thomas, M., Scott, T., 2011. Impact of daily temperature fluctuations on dengue virus transmission by Aedes aegypti, Proceedings of the National Academy of Sciences of the United States of America, 108, 18, 7460-7465.

Langlois, P., 2010. Simulation des systèmes complexes en géographie: fondements théoriques et applications, Paris, HermèsLavoisier.

Lourenço, J., Recker, M., 2013. Natural, persistent oscillations in a spatial multi-strain disease system with application to dengue. PLoS Computational Biology, 9, 10, e1003308.

Lwin, M., Vijaykumar, S., Fernando, O., Cheong, S., Rathnayake, V., Lim, G., Theng, Y., Chaudhuri, S., Foo, S., 2013. A 21st century approach to tackling dengue: Crowdsourced surveillance, predictive mapping and tailored communication, Acta Tropica, 130, 100-107.

Maciel-de-Freitas, R., Koella, J.C., Lourenco-de-Oliveira, R., 2011. Lower survival rate, longevity and fecundity of Aedes aegypti females orally challenged with dengue virus serotype 2, Transactions of the Royal Society of Tropical Medicine and Hygiene, 105, 452-458.

Magori, K., Legros, M., Puente, M., Focks, D., Scott, T., Lloyd, A., Gould, F. 2009. Skeeter Buster: A stochastic, spatially explicit modeling tool for studying Aedes aegypti population replacement and population suppression strategies, PLoS Neglected Tropical Diseases, 3, 9, e508, doi: 10.1371/ journal.pntd.0000508.

Maneerat, S., Daudé, É., 2015. An agent-based model of dengue vector, Aedes aegypti, to understand the spatiotemporal heterogeneity of mosquito populations, Ecological Modelling, à paraître.

Mpolya, E.A., Yashima, K., Ohtsuki, H., Sasaki, A., 2014. Epidemic dynamics of a vector-borne disease on a villagesand-city star network with commuters, Journal of Theoretical Biology, 343, 120-126.

OMS, 2012a. Global strategy for dengue prevention and control 2012-2020, Genève (online: http: / / www.who.int/denguecontrol/9789241504034/en/index.html).

OMS, 2012b. Handbook for clinical management of dengue, Genève (online: http://www.who.int/denguecontrol/ 9789241504713/en/).

Ooi, E., Goh, K., Gubler, D., 2006. Dengue prevention and 35 years of vector control in Singapore, Emerging Infectious Diseases, 12, 887-893.

Ordóñez-Gonzalez, J.G., Mercado-Hernandez, R., Flores-Suarez, A.E., Fernández-Salas, I., 2001. The use of sticky ovitraps to estimate dispersal of Aedes aegypti in Northeastern Mexico, Journal of American Mosquito Control Association, 17, 93-97.

$\mathrm{PAHO}, 1997$. Re-emergence of dengue in the Americas, Epidemiological Bulletin, 18, 2.

Palaniyandi, M., 2014. Web mapping GIS: GPS under the GIS umbrella for Aedes species dengue and chikungunya vector mosquito surveillance and control, International Journal of Mosquito Research, 1, 3, 18-25.

Picheral, H., 1983. Complexes et systèmes pathogènes: approche géographique. De l'épidémiologie à la géographie 
humaine, Travaux et Documents de Géographie Tropicale, 48, 5-22.

Rodhain, F., Rosen, L., 1997. Mosquito vectors and dengue virus-vector relationships, in Gubler, D., Kuno, G., (Ed.), Dengue and Dengue Haemorrhagic Fever, London, CAB International, 45-60.

Rogers, D., Randolph, S., 2006. Climate change and vectorborne diseases, Advances in Parasitology, 62, 345-381.

Rotela, C., Fouque, F., Lamfri, M., Sabatier, P., Introini, V., Zaidenberg, M., Scavuzzo, C., 2007. Space-time analysis of the dengue spreading dynamics in the 2004 Tartagal outbreak, Northern Argentina, Acta Tropica, 103, 1, 1-13.

Sabchareon, A., Wallace, D., Sirivichayakul, C., Limkittikul, K., Chanthavanich, P., Suvannadabba, S., Jiwariyavej, V., Dulyachai, W., Pengsaa, K., Wartel, T.A., Moureau, A., Saville, M., Bouckenooghe, A., Viviani, S., Tornieporth, N.G., Lang, J, 2012. Protective efficacy of the recombinant, live-attenuated, CYD tetravalent dengue vaccine in Thai schoolchildren: A randomized, controlled phase $2 \mathrm{~b}$ trial, Lancet, 380, 1559-1567.

Salje, H., Lessler, J., Endy, T., Curriero, F., Gibbons, R., Nisalak, A., Nimmannitya, S., Kalayanarooj, S., Jarman, R., Thomas, S., Burke, D., Cummings, D., 2012. Revealing the microscale spatial signature of dengue transmission and immunity in an urban population, Proceedings of the National Academy of Sciences of the United States of America, 109, 95359538.

Schmidt, W.-P., Suzuki, M., Thiem, V., White, R., Tsuzuki, A., Yoshida, L., Yanai, H., Haque, U., Tho, L., Anh, D., Ariyoshi, K., 2011. Population density, water supply, and the risk of dengue fever in Vietnam: cohort study and spatial analysis, PLoS Medecine, 8, e1001082, doi: 10.1371/journal.pmed.1001082.

Setbon, M., Raude, J., 2009. Population response to the risk of vector-borne diseases: lessons learned from sociobehavioural research during large-scale outbreaks, Emerging Health Threats Journal, 2, doi.org/10.3402/ehtj.v2i0. 7083.

Sharma, Y., Kaur, M., Singh, S., Pant, L., Kudesia, M., Jain, S., 2012. Seroprevalence and trend of dengue cases admitted to a government hospital, Delhi - 5-year study (2006-2010): A look into the age shift, International Journal of Preventive Medicine, 3, 8, 537-543.

Singh, R., Mittal, P., Yadav, N., Gehlot, O., Dhiman, R., 2011. Aedes aegypti indices and KAP study in Sangam Vihar, south Delhi, during the XIX Commonwealth Games, New Delhi, 2010, Dengue Bulletin, 35, 131-140.
Stoddard, S.T., Forshey, B., Morrison, A., Paz-Soldan, V., Vazquez-Prokopec, G., Astete, H., Reiner, R., Vilcarromero, S., Elder, J., Halsey, E., Kochel, T., Kitron, U., Scott, T., 2013. House-to-house human movement drives dengue virus transmission, Proceedings of the National Academy of Sciences of the United States of America, 110, 3, 994-999.

Tatem, A.J., Rogers, D., Hay, S.I., 2006. Global transport networks and infectious disease spread, Advances in Parasitology, 62, 293-343.

Telle, O., 2011. Le système indien de surveillance des maladies infectieuses face au risque denguien : croyances et actions de luttes sur les espaces endémiques, Espace Populations et Sociétés, 1, 47-62.

Thammapalo, S., Chongsuvivatwong, V., Geater, A., Dueravee, M., 2008. Environmental factors and incidence of dengue fever and dengue hemorrhagic fever in an urban area, Southern Thailand, Epidemiology and Infection, 136, 135-143, doi:10.1017/S0950268807008126.

Treuil, J.-P., Drogoul, A., Zucker, J.-D., 2008. Modélisation et simulation à base d'agents : exemples commentés, outils informatiques et questions théoriques, Paris, Dunod.

Tun-Lin, W., Lenhart, A., Nam, V., Rebollar-Tellez, E., Morrison, A., Barbazan, P., Cote, M., Midega, J., Sanchez, F., Manrique-Saide, P., Kroeger, A., Nathan, M., Meheus, F., Petzold, M., 2009. Reducing costs and operational constraints of dengue vector control by targeting productive breeding places: A multi-country non-inferiority cluster randomized trial, Tropical Medicine and International Health, $14,9,1143-1153$.

Vezzani, D., Rubio, A., Velazquez, S., Schweigmann, N., Wiegand, T., 2005. Detailed assessment of microhabitat suitability for Aedes aegypti in Buenos Aires, Argentina, Acta Tropica, 95, 123-131.

Watts, D., Burke, D., Harrison, B., Whitmire, R., Nisalak, A., 1987. Effect of temperature on the vector efficiency of Aedes aegypti for dengue 2 virus, The American Journal of Tropical Medicine and Hygiene, 36, 143-152.

Weaver, S., Vasilakis, N., 2009. Molecular evolution of dengue viruses: Contributions of phylogenetics to understanding the history and epidemiology of the preeminent arboviral disease, Infections, Genetics and Evolution, 9, 523-540.

Yang, H., Macoris, M., Galvani, K., Andrighetti, M., Wanderley, D., 2009. Assessing the effects of temperature on the population of Aedes aegypti, the vector of dengue, Epidemiology and Infection, 137, 8, 188-202. 